\title{
ENHANCED ROUTING PROTOCOLS FOR UWSN USING MRP TECHNOLOGY
}

\author{
Jaibheem $^{1}$, Mallikarjun Gokavi ${ }^{2}$, Kailash Patil ${ }^{3}$ \\ ${ }^{1}$ Student of ECE Dept (M.TECH), VNEC Shorapur \\ ${ }^{2}$ HOD Dept. of ECE, VNEC Shorapur \\ ${ }^{3} P G$ Coordinator ECE Dept VNEC Shorapur
}

\begin{abstract}
The Acoustic Signals Used As Physical Medium For Communication In Under Water Wireless Sensor Networks. Due To the Use of Acoustic Signals in UWSN, It Have The Distinguish Characteristics. The Different Types of Parameter in UWSN'S Are Including High Propagation Delay, Low Bandwidth High Error Rates and Significantly Different from Wireless Sensor Networks. Hence, Designing Communication Protocols, Particularly An Efficient Routing Protocol For UWSN Is A Challenging Issue. Routing Protocols Can Take Advantage Of The Localization Of Sensor Nodes. However, The Localization Itself Is Not Impeccable In UWSN. In This Paper, Therefore Propose An Enhanced Routing Protocol Named MRP (Multi-Layered Routing Protocol) For UWSN. MRP Utilizes Super Nodes In Order To Eliminate The Need Of Localization. MRP Works In Two Phases: Layering Phase And Data Forwarding Phase. During Layering Phase, Different Layers Are Formed Around The Super Nodes. In Data Forwarding Phase, Data Packets Are Forwarded Based On These Layers. Through Simulation Study Using MATLAB Simulator, To Prove That MRP Contributes Significant Performance Improvements Against A Deputy Routing Protocols.
\end{abstract}

Keywords: Underwater Wireless Sensor Networks, Routing, Multilayer, Network Lifetime, Delivery Ratio, Residual Energy.

\section{INTRODUCTION}

Underwater Wireless Sensor Networks (UWSNs) have attracted much research attention due to their various applications for scientific, environmental, commercial and military purposes.

In UWSNs, radio and optical signals are known to incur poor performance. The radio sig-nals propagate at long distances only at extra low frequencies (30-300 Hz), which requires large antennas and high transmission power. Optical signals are affected from scattering and require high precision in pointing the narrow laser beams [ 3] However, the employment of acoustic signals imposes many distinctive challenges on UWSNs i.e. large propagation delay of acoustic signals (i.e. $1,500 \mathrm{~m} / \mathrm{s}$ ), limited bandwidth (i.e. $<100 \mathrm{KHz}$ ), and high bit error rates due to the extreme characteristics of the underwater channel [12]. Furthermore, the sensor nodes in UWSNs contain limited energy [ 13]. The replacement of batteries of such nodes is very expensive due to harsh underwater environment. Hence, prolonging the network lifetime in such networks like UWSNs in very important $[4,14,15]$. Therefore propose a localization-free routing protocol for UWSNs named MRP (Multi-layered Routing Protocol). MRP utilizes super nodes for routing. The super nodes are the nodes having abundant energy and have the capability of transmitting a packet with different transmission powers.

In this paper, the super nodes are deployed at various depths from the top to the bottom of the deployment region. The super nodes at the highest depth collect data from the ordinary sensor nodes and transmit it towards sink by relaying it through the super nodes available at lower depths. The ordinary sensor nodes forward the data packets to the super node based on a layering structure. The involvement of lim-ited number of nodes during forwarding reduces the energy consumption. Furthermore, each forwarding node in our proposed protocol do not wait unnecessarily before forwarding the data packet and the super nodes having high transmission powers participate in forwarding, which leads to very short end-to-end delay.

\section{RELATED WORK}

Localization Based Routing Protocols: the routing protocols that are based on localization of sensor nodes. In[16], VBF (vector based forwarding) protocol was proposed. In VBF, the source node computes a vector from itself towards the destination/sink node. The nodes residing in the area around the computed vector, up to a certain radius, are allowed to participate in forwarding. it has some drawback that in case of sparse density, the unavailability of the nodes in the routing pipe affects the forwarding. Furthermore, the hard assumption of the localization is another factor to determine its performance. In [ 17], an extension of VBF protocol called HHVBF (hop-by-hop $\mathrm{VBF}$ ) was proposed. In HHVBF, the vector is computed on per-hop basis i.e. each forwarding node computes a vector from itself towards the sink. The re-computation of the vector at each hop reduces the effect of the sparse density problem. FBR (focused beam routing) protocol [18] uses 
different transmission powers during the selection of a forwarding node. According to the routing process, a sender transmits an RTS packet with a certain transmission power. If a CTS packet is received, the data packet is transmitted to the sender of the CTS packet. FBR suffers from the increased delay due to the exchange of RTS/CTS packets as well as the assumption of the localization of the source and sink node.

Localization-Free Routing Protocols: In [ 19], DBR (depth based routing) protocol was presented. DBR utilizes the depth of the sensor nodes as a routing metric. The sender of the data packet includes its depth in the data packet. The receiving nodes compare their depths with the depth of the sender and the nodes having lower depths further forward the data packet. , the forwarding of data packets based on only the depth of sensor nodes has some limitations. First, as the network density increases, the number of redundant packet transmissions also increases. Second, it has the unbalanced energy consumption during data forwarding. Since the depth of sensor nodes is the only parameter for forwarding, the nodes having smaller depths forward the data packets most of the time. Therefore, the energy of such nodes is exhausted soon, and these nodes die earlier than the other nodes. H2-DAB (hop by hop dynamic addressing based) routing protocol [20] assigns a unique ID called a HopID to each sensor node based on hop count towards the sink node. The HopID is assigned using Hello messages broadcasts by the sinks residing at the water surface. Each receiving sensor node is assigned a HopID. These sensor nodes then re-broadcast the Hello packet after incrementing the HopID. This process is repeated until all the nodes in the network are assigned their HopID.

Winston et al. [ 21] proposed a scheme where multiple sink nodes are assumed connected with each other. Similar to H2-DAB, each sensor node is assigned a hop count value. During the forwarding of data packets, these hop count values are used as a routing metric for forwarding the data packets.

\section{MRP: MULTI-LAYERED ROUTING}

\section{PROTOCOL}

MRP (multi-layered routing proto-col), in detail with proposed network architecture, layering and data forwarding techniques.

Network Architecture: As shown in Fig. 1, sinks are deployed at the water surface, where these sinks are connected to each other through radio links. Sensor nodes are deployed underwater from the top to the bottom of the deployment region at various depths. Sensor nodes send data packets to sink by relaying them through the neighbouring sensor nodes residing closer to sink. Particularly, in UWSNs, the sensor nodes residing at higher depths forward the data packets by relaying them through the sensor nodes at lower depths. Hence, the communication occurs from the bottom towards the top/surface of the water. Figure 2 shows our proposed network architecture. We have utilized two different types of nodes, i.e. super nodes and ordinary sensor nodes. Super nodes are the nodes having abundant energy and having the capability of transmitting a packet with various transmission powers.

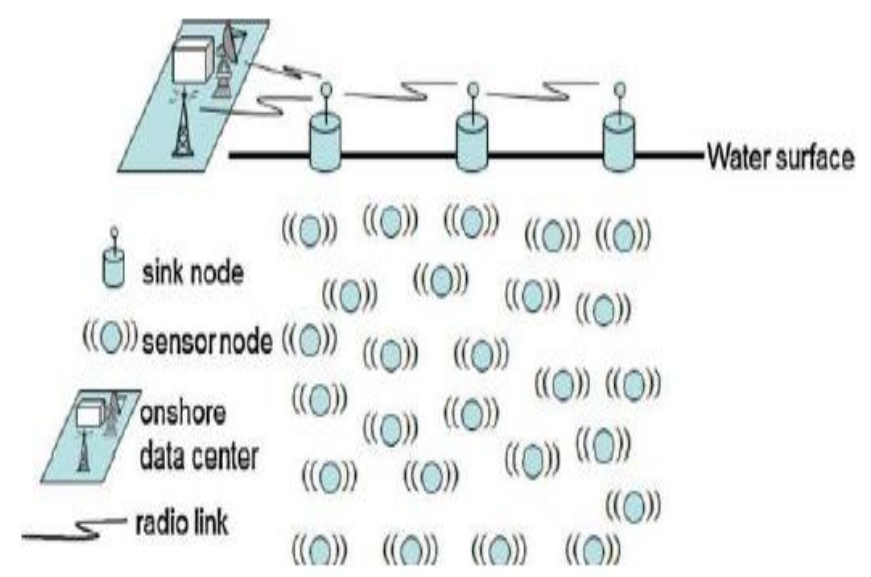

Fig. 1 A typical architecture of an underwater wireless sensor network

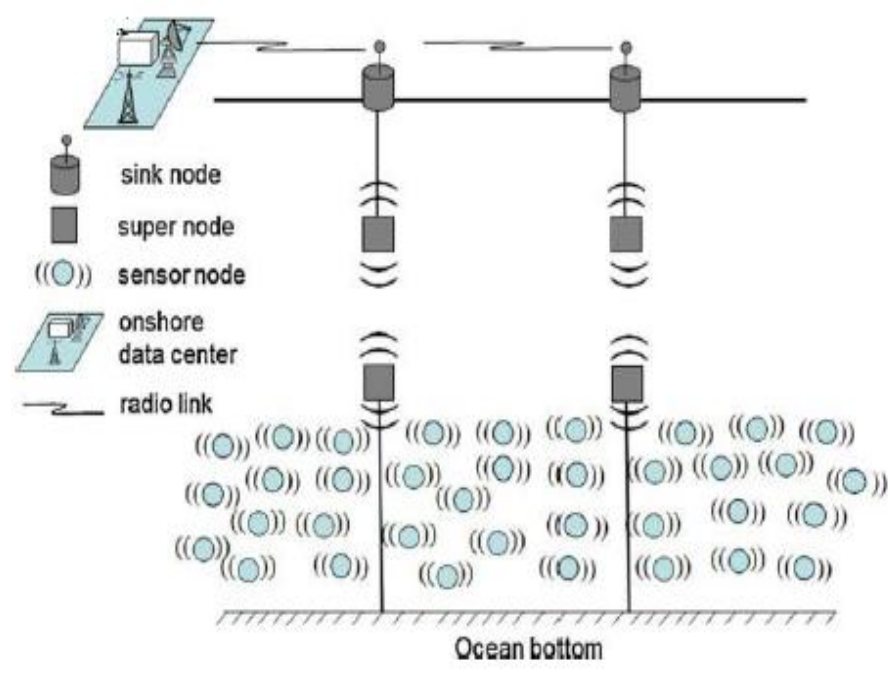

Fig. 2 our proposed network architecture

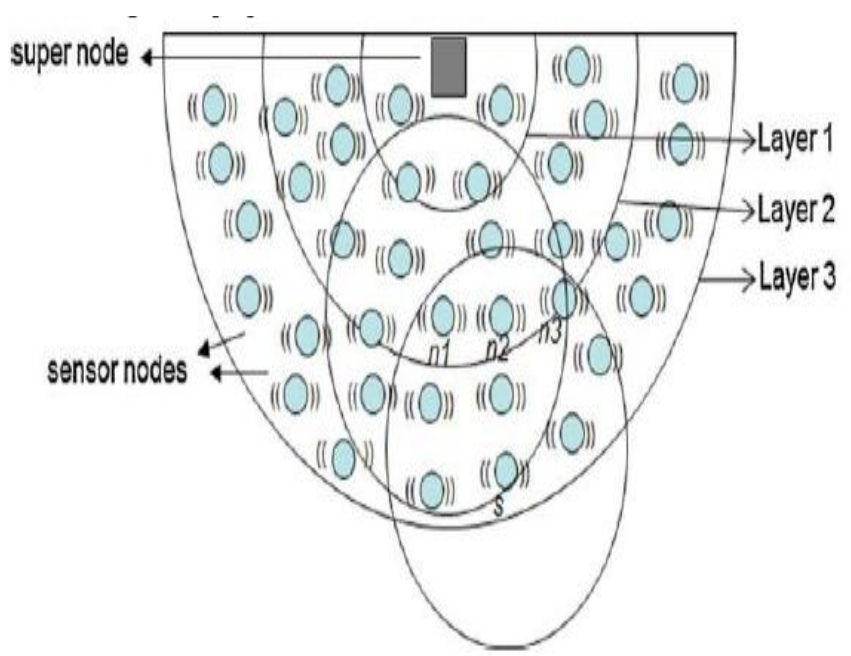

Fig. 3 An illustration of a forwarding node selection 
Performance Evaluation: , MRP. The performance of MRP was compared against a well-known localization-free routing protocol DBR [ 19] and another localization-free routing protocol H2-DAB [ 20]. We also compared MRP with a representative localization-based routing protocol VBF [ 16].
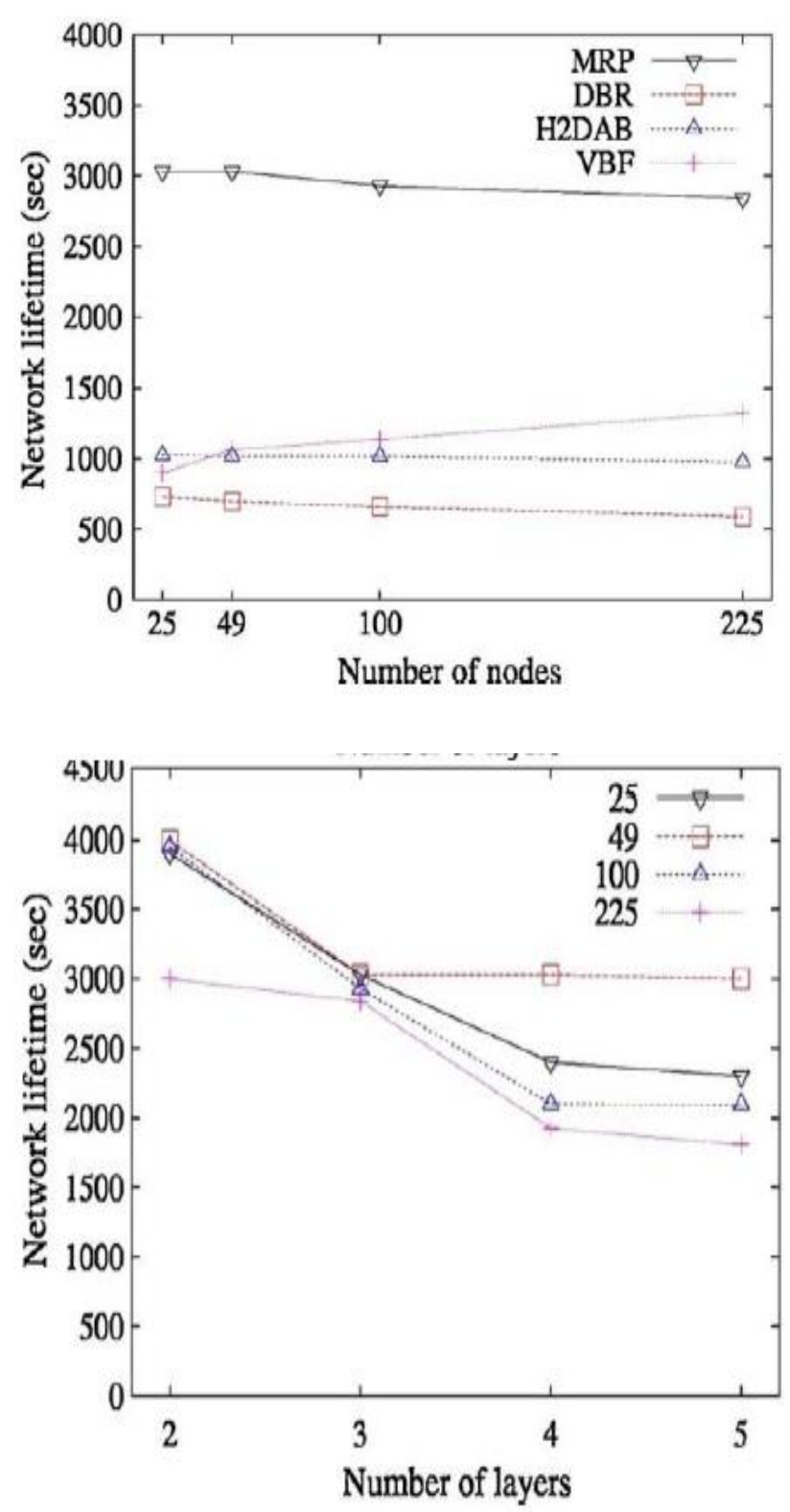

Fig. 4 Network lifetime against DBR, H2DAB and VBF
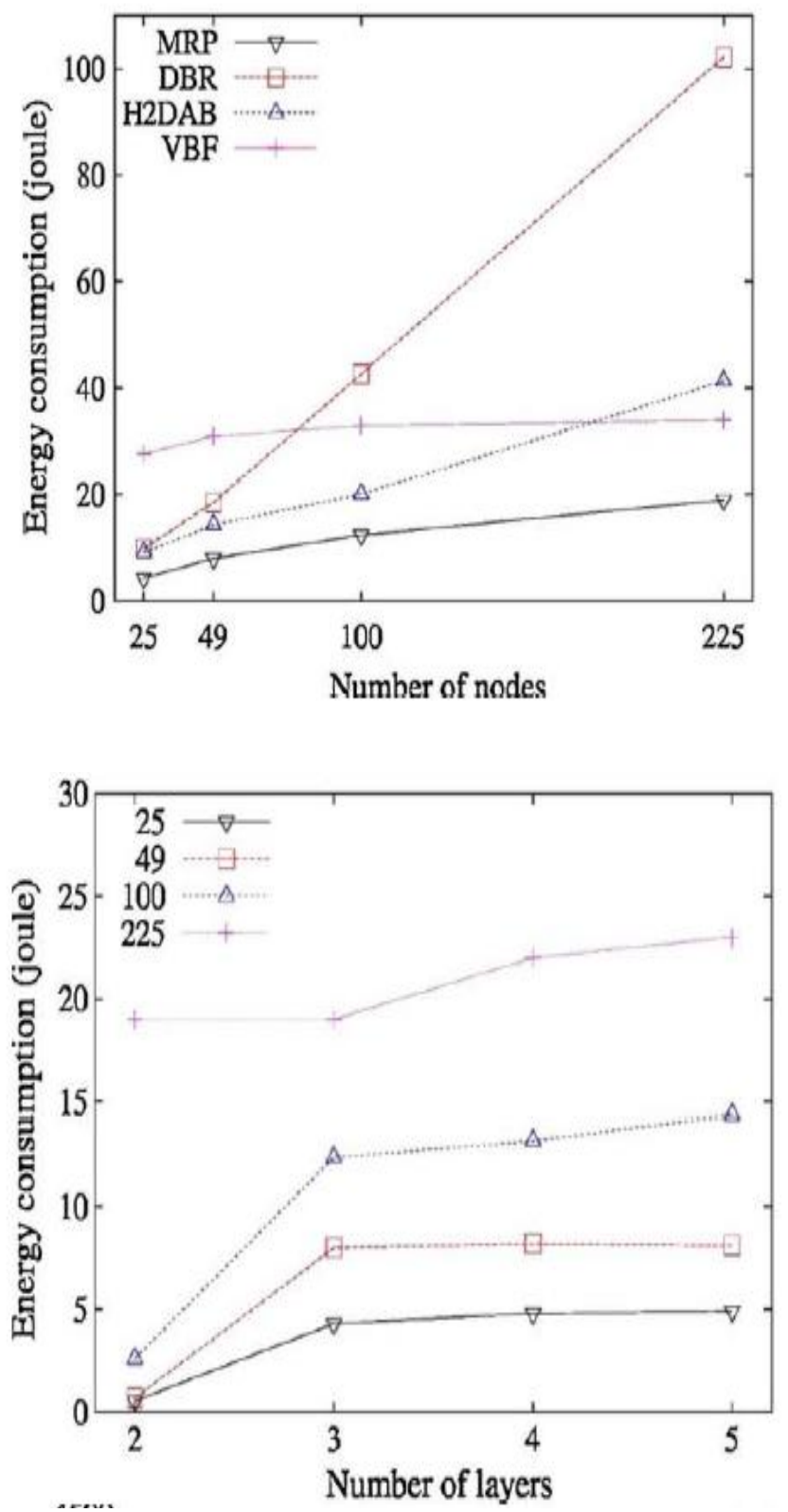

Fig. 5 Energy consumption against DBR, H2DAB and VBF

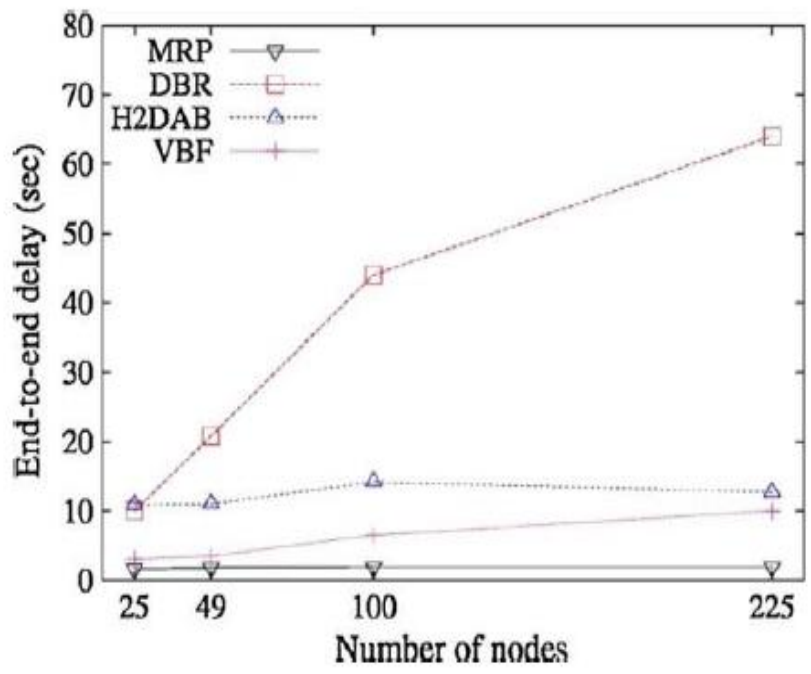

Fig. 6 End-to-end delay againstDBR, H2DAB and VBF 

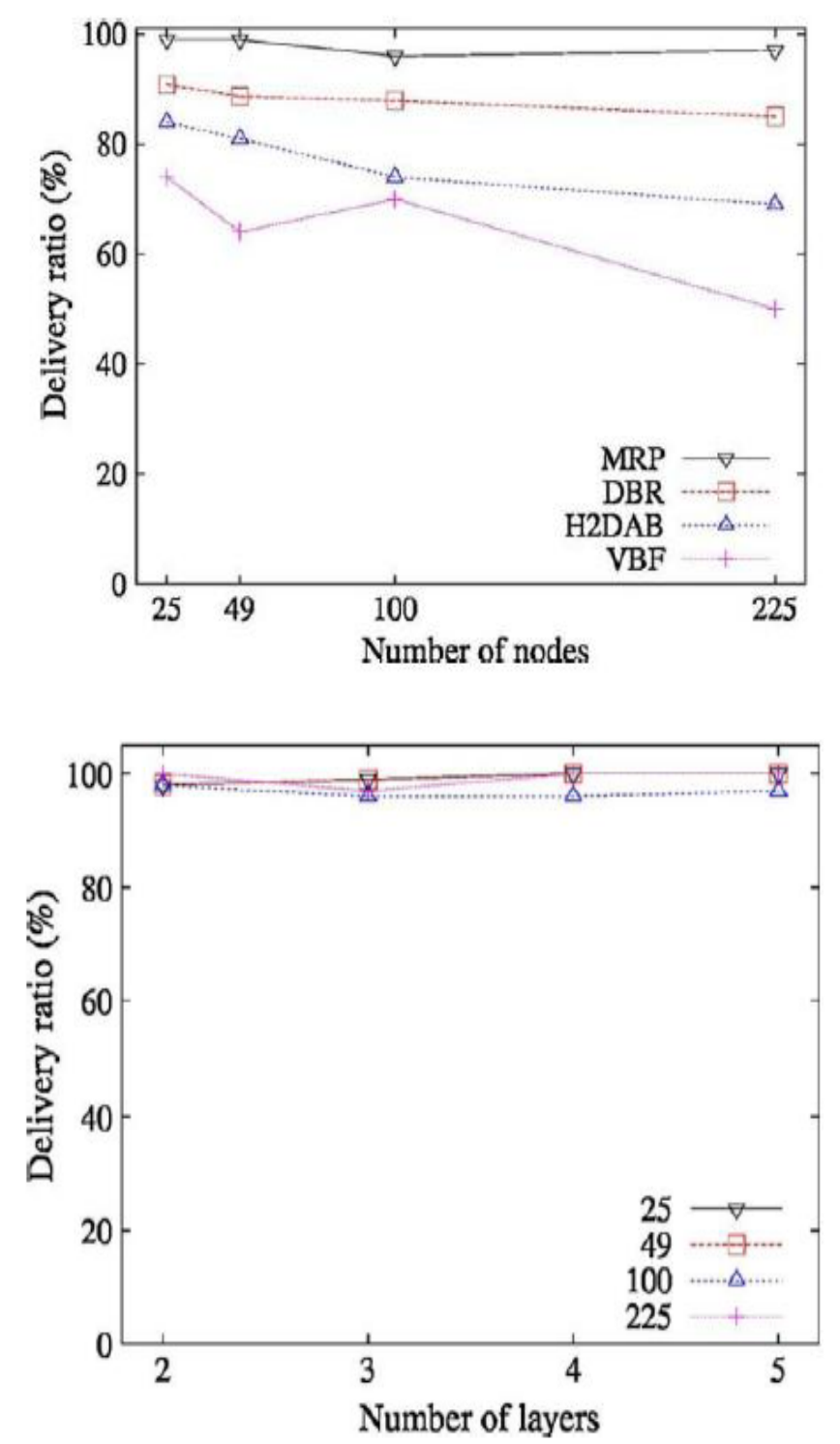

Fig. 7 Delivery ratio against DBR, H2DAB and VBF

Simulation Results with Different Number of Layers: simulation results of MRP protocol with different number of layers. The number of layers is an important parameter in MRP since number of layers affects the different parameters i.e. network lifetime, energy consumption Delivery ratio with different number of layers Respectively.

\section{CONCLUSION}

In this paper, proposed a localization-free routing protocol named MRP (multi-layered routing protocol) for UWSNs. MRP uses super nodes in order to eliminate the need of localization of the sensor nodes. Super nodes are the nodes having abundant energy and have the ability of transmitting packets using different transmission powers. MRP works in two phases: Layering phase and Data forwarding phase. During layering phase, different layers are formed around the super nodes, and each sensor node is assigned an ID called Layer ID. During data forwarding phase, data packets are forwarded on the basis of the assigned Layer IDs. The source node forward the data packet towards the super nodes deployed at the highest depths. These super nodes then forward data packets towards sinks by relaying them. MRP is compared against well-known localization-free routing protocols DBR [ 19], H2DAB [ 20] and a localization-based routing protocol VBF [ 16]. We observed that MRP has improved performance in terms of network lifetime, energy consumption, end- to-end delay and delivery ratio. The network lifetime of MRP is 75-79, 65-66, 53-70 \% better than DBR, H2DAB and VBF, respectively. Energy consumption of MRP shows 56-81, 38-54 and 44-84\% better performance than DBR, H2DAB and VBF, respectively. Also, the end-to-end delay is 84-97, 83-86 and 48-81\% lower than DBR, H2DAB and VBF, respectively. MRP also has 8-12, 15-28, and 25-48 \% better delivery ratio against $\mathrm{DBR}, \mathrm{H} 2 \mathrm{DAB}$, and $\mathrm{VBF}$, respectively. We have also simulated our proposed MRP routing protocol using different number of layers. It was observed that number of layers affects the performance of proposed protocol MRP. Therefore, a proper selection of number of layers is important for the performance of MRP.

\section{REFERENCES}

[1] Abdul Wahid - Sungwon Lee · Dongkyun Kim · Kyung-Shik Lim(2014), A Localization-Free MultiLayered Routing Protocol for Underwater Wireless Sensor Networks Wireless Pers Commun (2014) 77:2997-3012 DOI 10.1007/s11277-014-1690-6

[2] Wafta, M. K., Selman, S., \& Denkilkian, H. (2010). UW-MAC: An underwater sensor network MAC protocol. International Journal of Communication Systems, 23, 485-506. doi: 10.1002/dac.1086.

[3] Babu, A. V., \& Joshy, S. (2012). Maximizing the data transmission rate of a cooperative relay system in an underwater acoustic channel. International Journal of Communication systems, 25, 231-253. doi: 10. 1002/dac.1266.

[4] Akylidiz, I. F., Pompili, D., \& Melodia, T. (2005). Underwater acoustic sensor networks: Research challenges. Ad Hoc Networks, 3(3), 257-279.

[5] Zhang, S., Li, D., \& Chen, J. (2013). A link-state based adaptivefeedback routing for underwater acoustic sensor networks. IEEESensors Journal, 13, 4402-4412.

[6] Filipe, L., \& Vieira, M. (2012). Performance and trade-offs ofopportunisic routing in underwater networks. In Proceedings of IEEE Wireless Communication and Neworking Conference (WCNC), Shanghai, China, 1-4 April 2012 (pp. 2911-2915). doi: 10.1109/WCNC.2012.6214301.

[7] Ren, Y., Seah, W. K. G., \& Teal, P. D. (2012). Performance of pressure routing in drifting 3D underwater sensor networks for deep water monitoring. In Proceedings of the seventh ACMinternational conference on underwater networks and systems. doi: 10.1145/2398936.2398972.

[8] Chen, Y.-S., \& Lin, Y.-W. (2013). Mobicast routing protocol for underwater sensor networks. IEEE Sensors Journal, 13(2), 737-749. doi: 10.1109/JSEN.2012.2226877. 
[9] Cai, S., et al. (2013). A network coding based protocol for reliabledata transfer in underwater acoustic sensor. Ad Hoc Networks, 11(5), 1603-1609.

[10] Tsai, C. S., \& Yang, C. F. (2013). A novel energy efficient joint dynamic emissive location-based routing scheme for SOFAR channel underwater sensor networks. Applied Mechanics and Materials, 284-287(7), 2001-2004.

[11] Wahid, A., \& Kim, D. (2012). Connectivity-based routing protocol for underwater wireless sensor networks. In Proceedings of IEEE ICT Convergence (ICTC), Jeju Island, Korea, 15-17 October 2012 (pp. 589-590). doi: 10.1109/ICTC.2012.6387207.

[12] Harris, A. F., \& Zorzi, M. (2007). Modeling the underwater acoustic channel in ns2. In Proceedings of the 2nd international conference on performance evaluation methodologies and tools (ValueTools'07).

[13] Chen, K., Zhou, Y., \& He, J. (2009). A localization scheme for underwater wireless sensor networks.

[14] International Journal of Advanced Science and Technology, 4, 9-16.

[15] Dhurandher, S. K., Obaidat, M. S., \& Gupta, M. (2012). Providing reliable and link stability-based geocasting model in underwater environment. International Journal of Communication Systems, 25, 356-375. doi: 10.1002/dac.1245.

[16] Son, J., \& Byun, T.-Y. (2011). A routing scheme with limited flooding for wireless sensor networks.

[17] International Journal of Future Generation Communication and Networking, 3(3), 19-31. 